\title{
An Ultrastructural Study of Isolated Rat Skeletal Muscle Mitochondria in Various Metabolic States
}

\author{
JERRY M. KUNER and ROBERT E. BEYER \\ Laboratory of Chemical Biology and Department of Zoology, University of Michigan, \\ Ann Arbor, Michigan 48104
}

Received 22 September 1969

Summary. An electron microscopic examination was made of isolated rat skeletal muscle mitochondria in various functional states. Shifts in the inner membrane ultrastructure of populations of mitochondria were observed under certain conditions. However, ultrastructural transformations reported by others during rapid changes in biochemical states were not observed in skeletal muscle mitochondria. There does not appear to be a strict correlation between metabolic states and ultrastructural states in isolated rat skeletal muscle mitochondria as has been observed in isolated mitochondria from several other tissue types.

A correlation between the ultrastructural and biochemical states of isolated mitochondria has been reported from a number of laboratories $[4-8,13]$. Hackenbrock [6], utilizing rat and mouse liver mitochondria, has demonstrated reversible ultrastructural transformations of the inner mitochondrial membrane during biochemical transitions from state 4 to state 3 respiration as defined by Chance and Williams [1]. He has identified two primary ultrastructural configurations, condensed and orthodox, associated with states 3 and 4, respectively. Goyer and Krall [4] have reported similar results with rat kidney mitochondria. Using beef heart mitochondria, Green and his colleagues $[5,8]$ have identified a number of ultrastructural states associated with functional states and have postulated an energy-conservation scheme based on configurational changes in the inner mitochondrial membrane. These latter authors [8] postulate that the conformational changes of individual components of the inner mitochondrial membrane result in a configurational change in the entire membrane which is visible with the use of the electron microscope.

Recently, Sordahl, Blailock, Kraft, and Schwartz [15] failed to find a generalized relationship between ultrastructural and biochemical states in heart mitochondria isolated from a variety of species. Ultrastructural 
transformations that are the basis of hypotheses to explain energy conservation during oxidative phosphorylation should be demonstrated in as wide a variety of tissue sources as possible. Therefore, an ultrastructural study was undertaken on rat skeletal muscle mitochondria routinely available in this laboratory.

\section{Materials and Methods}

Mitochondria from hind-leg skeletal muscles of adult male Sprague-Dawley rats (approximately $300 \mathrm{~g}$ ) were isolated by the method of Makinen and Lee [11], the final isolation medium being $0.25 \mathrm{M}$ sucrose. Mitochondrial protein was determined by a biuret procedure [3] after solubilization of particulate matter with deoxycholate. Oxygen consumption was determined with a Clark electrode in a 1.5 -ml water-jacketed glass cell maintained at $25^{\circ} \mathrm{C}$. Signals from the Clark electrode were monitored as described by Estabrook [2] with a Heath EUW-20 M servo-recorder. Dissolved oxygen was assumed to be $240 \mu \mathrm{M}$ at $25^{\circ} \mathrm{C}$. The various functional states were induced in a medium consisting of $0.15 \mathrm{M}$ sucrose, $25 \mathrm{~mm}$ Tris- $\mathrm{HCl}, 6 \mathrm{mM} \mathrm{K}_{2} \mathrm{HPO}_{4}$, and $5 \mathrm{~mm} \mathrm{MgSO}_{4}$, pH 7.5 . Several experiments were also performed in an incubation medium consisting of $0.112 \mathrm{M}$ $\mathrm{KCl}, 0.01 \mathrm{M}$ potassium phosphate buffer, pH $7.5,5 \mathrm{~mm} \mathrm{MgCl}$, and $1 \mathrm{~mm}$ ethylenediaminetetraacetate. The results of the smaller number of experiments performed in the $\mathrm{KCl}$ medium were consistent with results of experiments reported below for the sucrose-containing medium. The various functional states were induced in the mitochondrial populations by the addition of various reagents via polyethylene tubes fitted to $\mu$ liter syringes. Samples $(100 \mu$ liter) of mitochondrial suspensions (containing approximately $0.09 \mathrm{mg}$ mitochondrial protein) were withdrawn from the reaction cuvette at appropriate times for preparation for electron microscopy. The conditions under which samples were taken are fully described in Results.

The mitochondrial samples were prepared for electron microscopy utilizing a technique described by Hackenbrock [6]. After removal from the reaction cuvette, the $100-\mu$ liter samples were transferred quickly to $200-\mu$ liter polyethylene centrifuge tubes and centrifuged at approximately $10,800 \times g$ for $30 \mathrm{sec}$ in a Coleman model 6-8111 centrifuge (this centrifuge accelerates to its maximum speed in approximately $2 \mathrm{sec}$ ). The supernatant solution was removed and quickly replaced with fixative. The fixative was normally $2 \%$ osmium tetroxide in $0.15 \mathrm{~m}$ sucrose $-25 \mathrm{~mm}$ Tris- $\mathrm{HCl}, \mathrm{pH} 7.5$. For the samples incubated in the sucrose isolation medium, the fixative was $2 \%$ osmium tetroxide in $0.25 \mathrm{M}$ sucrose $-25 \mathrm{~mm}$ Tris- $\mathrm{HCl}, \mathrm{pH} 7.5$. The period between sampling and fixation was approximately $40 \mathrm{sec}$.

The mitochondrial sample was maintained in the fixative for $1 \mathrm{hr}$ at $0^{\circ} \mathrm{C}$. The mitochondrial pellets were then removed and dehydrated in increasing concentrations of ethanol. The dehydrated pellets were embedded in a soft epoxy-resin mixture consisting of 4 parts Epon 812 (Shell), 10 parts dodecenylsuccinic anhydride, and 0.32 parts 2,4,6-tri(dimethylaminomethyl)phenol (DMP-30). The blocks were sectioned with glass knives on a Porter-Blum ultramicrotome. Sections were picked up on 300-mesh, uncoated copper grids (Fullam). They were stained in $4 \%$ uranyl acetate in $40 \%$ ethanol for $40 \mathrm{~min}$ at room temperature.

Electron micrographs were obtained on DuPont Cronar Ortho A Litho COA7 film with an RCA-EMU 4 electron microscope operating at $50 \mathrm{KV}$.

Analytical reagent grade chemicals were used whenever available; filtered, deionized, glass-distilled water was employed for all solutions. 


\section{Results}

An oxygen-electrode tracing representative of the mitochondria used in this investigation is shown in Fig. 1. The $\mathrm{RMM}^{1}$ displayed ADP/O ratios of 2.2 to 2.8 and RCI values of approximately 7 to 10 . The oxygen consumption rates were approximately 240 and 34 natoms oxygen/min per $\mathrm{mg}$ protein for states 3 and 4, respectively. All mitochondrial preparations were tested in this respect, and those preparations exhibiting an RCI lower than 7 were discarded. In addition, the RCI of all mitochondrial samples were tested, whenever possible, at the end of the particular experimental condition. Except in the case of the effect of oxidative phosphorylation uncoupler additions, the high degree of respiratory control was maintained.

As a control, RMM were incubated in the $0.25 \mathrm{M}$ sucrose isolation medium at $25^{\circ} \mathrm{C}$, and samples were withdrawn and fixed after various periods of incubation in the oxygen-electrode cuvette. After $5 \mathrm{~min}$ of such incubation, the mitochondria were predominantly in a configuration which will be termed condensed (Fig. 2A) after the terminology of Hackenbrock

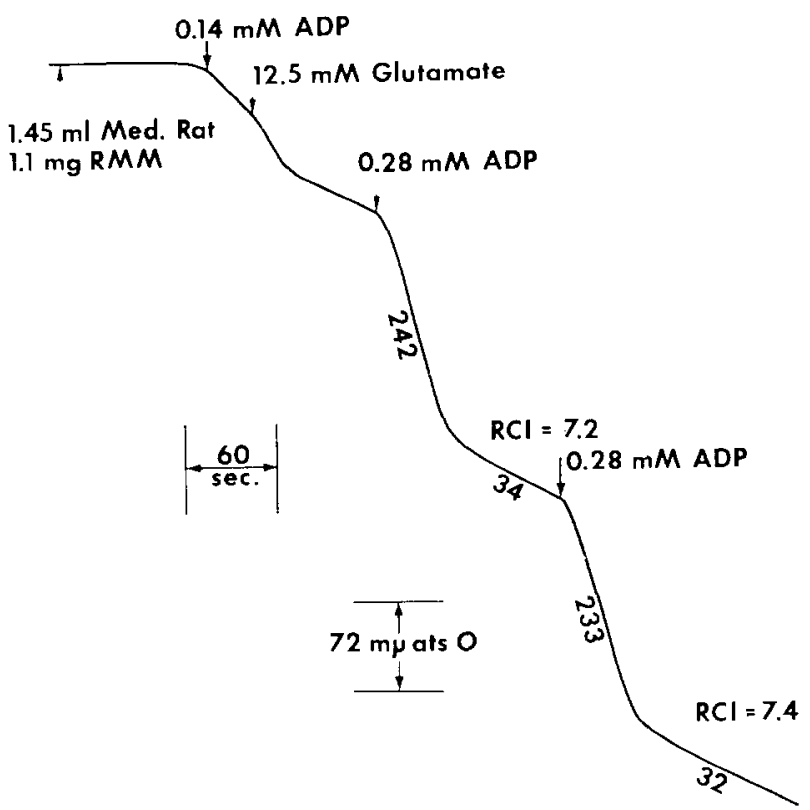

Fig. 1. Representative oxygen-electrode tracing of glutamate oxidation catalyzed by RMM. Numbers beneath the tracing refer to rates of oxidation in natoms/min per $\mathrm{mg}$ mitochondrial protein. Temperature: $25^{\circ} \mathrm{C}$

1 Abbreviations used are: $\mathrm{RMM}$, rat muscle mitochondria; $\mathrm{ADP} / \mathrm{O}$, ratio of nmoles ADP utilized to natoms oxygen consumed, a measure of the efficiency of oxidative phosphorylation; RCI, respiratory control index obtained by dividing the rate of oxygen consumption during state 3 by the rate of oxygen consumption in state 4 ; DNP, 2,4-dinitrophenol. 

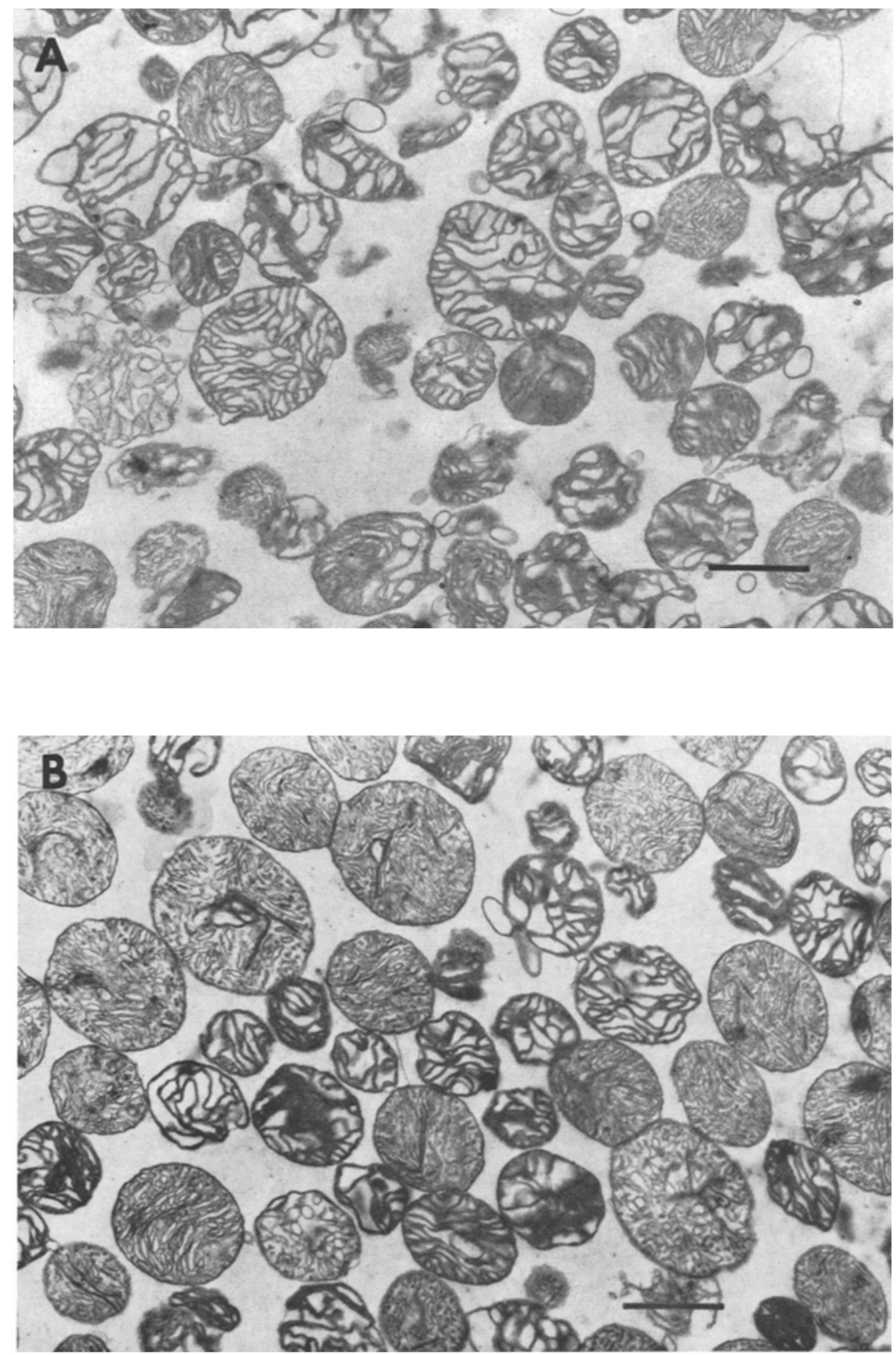

Fig. 2A and B. RMM sampled after 5-min (A) and 20-min (B) incubation in $0.25 \mathrm{M}$ sucrose. Note that most of the mitochondria in the 5-min sample are in the condensed configuration, whereas approximately $50 \%$ of the mitochondria have shifted to the orthodox configuration after the $20-\mathrm{min}$ incubation. Scale: $1 \mu . \times 16,000$ 

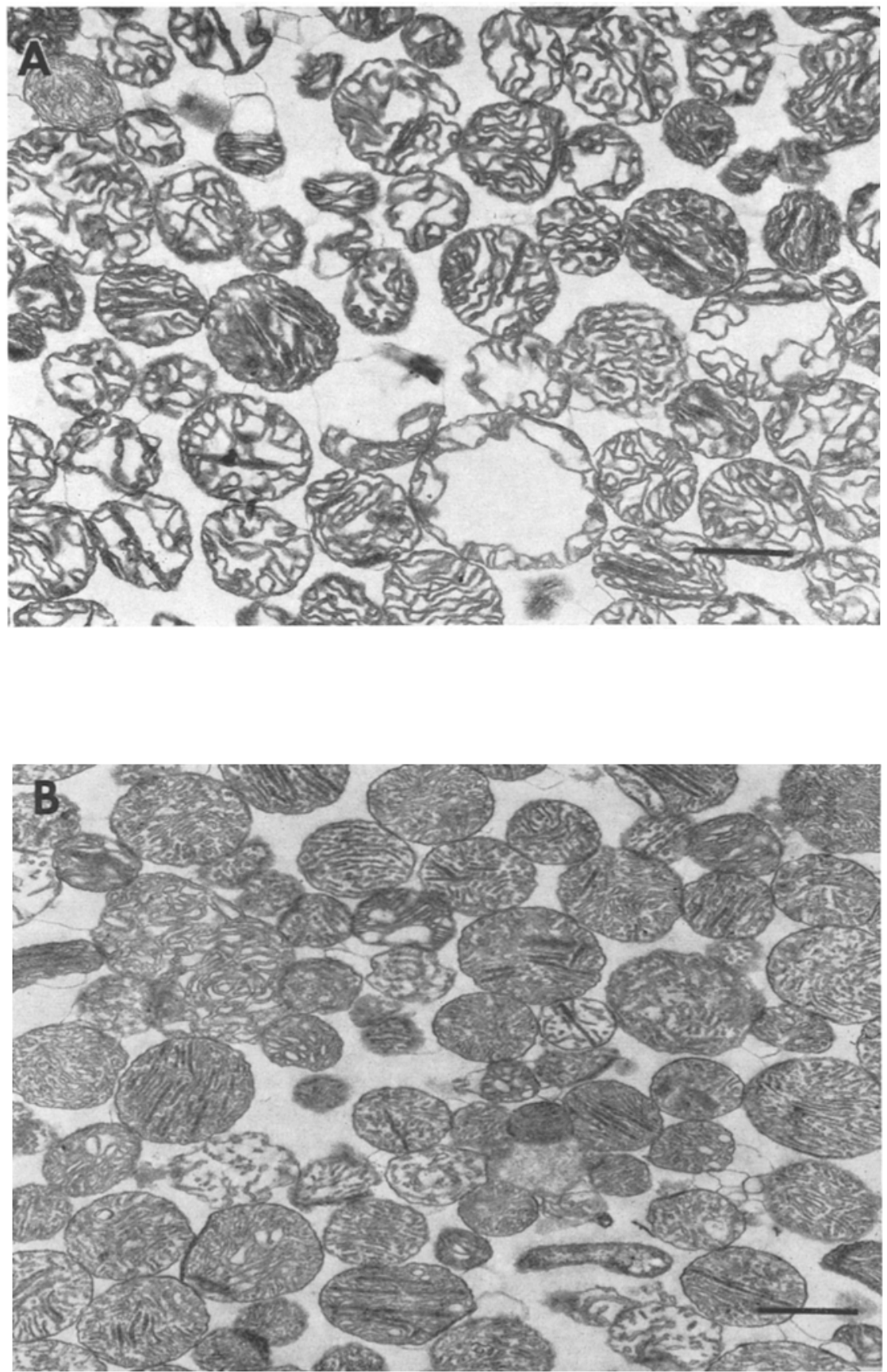

Fig. 3A and B. RMM sampled after 1-min (A) and 10-min (B) incubation in metabolic state 1 . Note that essentially all of the mitochondria have shifted from a highly condensed state at the 1-min incubation (A) to an essentially complete orthodox form after $10 \mathrm{~min}$ of incubation (B). Scale: $1 \mu . \times 16,000$ 


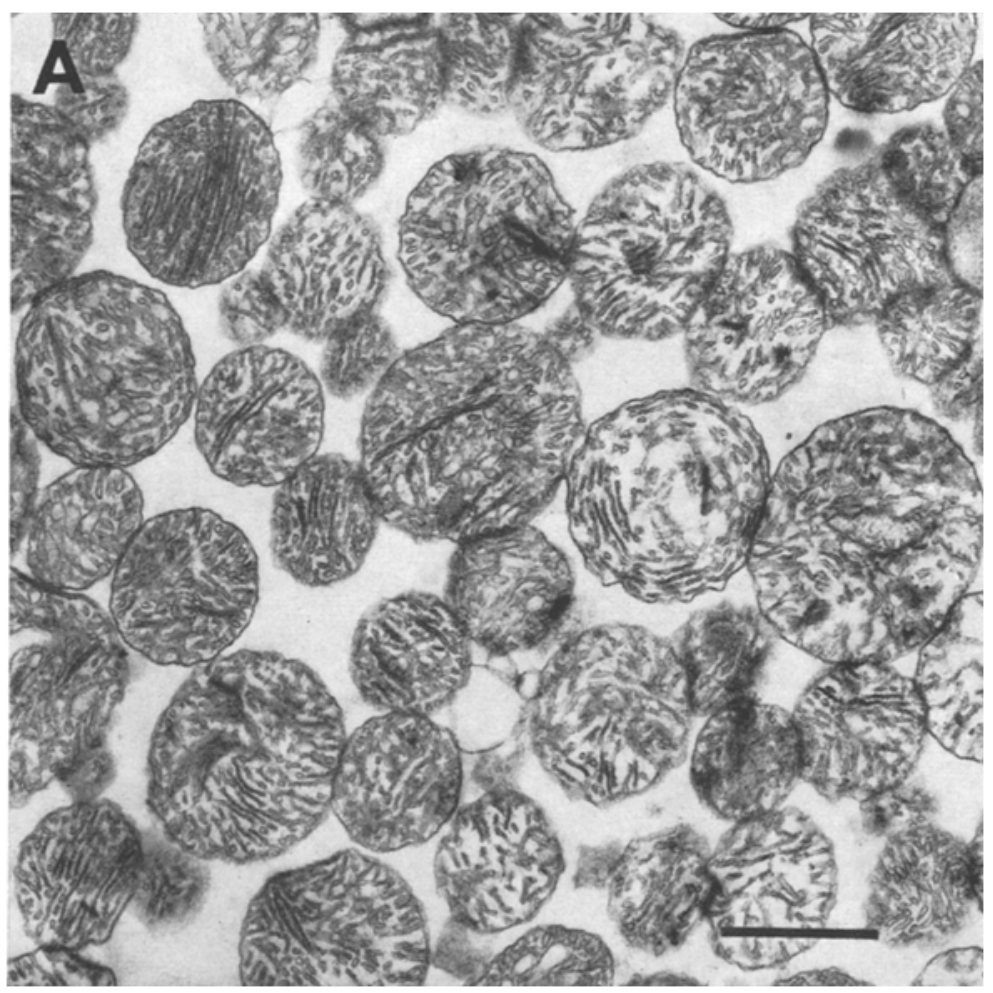

Fig. 4A-D. The effect of ADP, glutamate, ATP, or DNP on RMM incubated for $10 \mathrm{~min}$ in state 1 . Mitochondria were fixed immediately following the addition noted. Note that the mitochondria remained in the orthodox configuration after the addition of ADP to $0.14 \mathrm{~mm}(\mathrm{~A})$, glutamate to $12.5 \mathrm{~mm}(\mathrm{~B})$, ATP to $0.67 \mathrm{~mm}(\mathrm{C})$, or DNP to $10^{-4}$ M (D). Scale: $1 \mu$. $\times 16,000$

[6]. This configuration is characterized by large electron-transparent intracristal spaces and a highly compact electron-dense matrix. After 20-min incubation in $0.25 \mathrm{M}$ sucrose, approximately half of the RMM were in an orthodox configuration (Fig. 2B) characterized by well-defined cristae, small intracristal spaces, and a relatively dilute matrix [6].

State 1: RMM sampled after 1-min incubation in state 1 were virtually all in the condensed configuration (Fig. 3 A). After $10 \mathrm{~min}$ in state 1, all of the mitochondria appeared in the orthodox configuration (Fig. 3B). The addition of ADP (0.14 mm, Fig. 4A), glutamate (12.5 mM; Fig. 4B), ATP (0.67 mM; Fig. 4C), or DNP (0.1 mM; Fig. 4D) had no observable effect on the inner membrane configurations of the RMM. State 2: RMM placed in state 2 by the addition of ADP $(0.14 \mathrm{mM})$ to the reaction cuvette were in the condensed state after $1 \mathrm{~min}$ (Fig. 5A) and remained so after $20 \mathrm{~min}$ of 


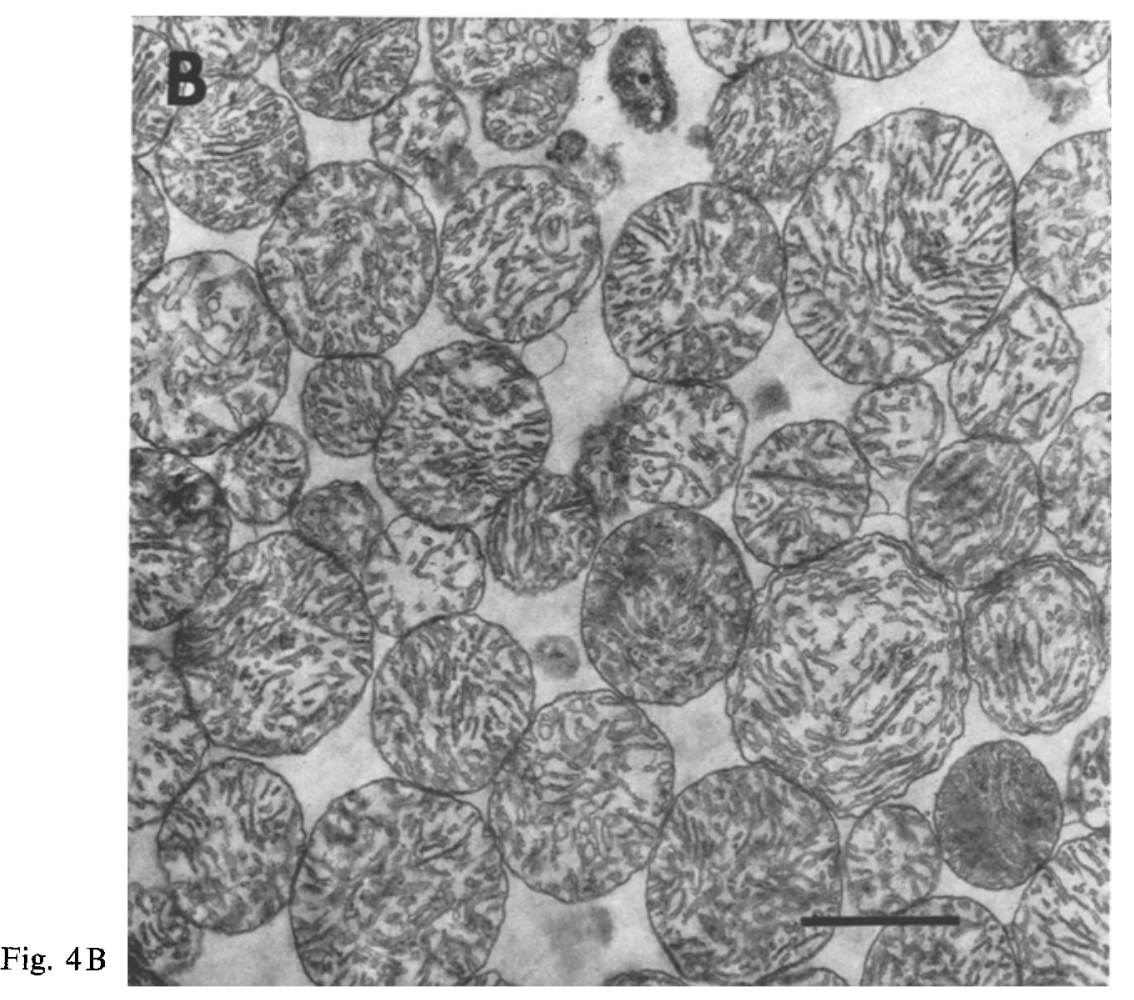

incubation (Fig. 5B). States 3 and 4: RMM were placed in state 4 (i.e., control of respiration after depletion of ADP by the reactions of oxidative phosphorylation) by adding ADP $(0.14 \mathrm{~mm})$ and glutamate $(12.5 \mathrm{~mm})$ to the reaction mixture and waiting for phosphorylation during state 3 to terminate. Air was bubbled into the reaction mixture during the state 4 incubation to prevent anaerobiosis in the mitochondrial suspension. After 5-min incubation in state 4, RMM were all in the condensed state (Fig. 6A) and did not change significantly even after 30-min incubation (Fig. 6B). Samples taken immediately after induction of state 3 by the addition of ADP $(0.42 \mathrm{~mm})$ or of state $3 \mathrm{U}$ by the addition of DNP $(0.1 \mathrm{~mm})$ indicated that these reagents had no observable effect on the membrane configurations of the RMM (Fig. 6C and D). That these same additions had profound effects on the metabolic state of the mitochondria is illustrated in Fig. 6E which shows the increase in respiration upon the addition of ADP and DNP to produce states 3 and $3 \mathrm{U}$, respectively. In addition, ATP was depleted by the addition of excess hexokinase and $50 \mu$ moles of glucose. Respiration immediately increased by a factor of 7.3, and the mitochondria were found to be in the condensed state, i. e., indistinguishable from those shown in Fig. 6. 

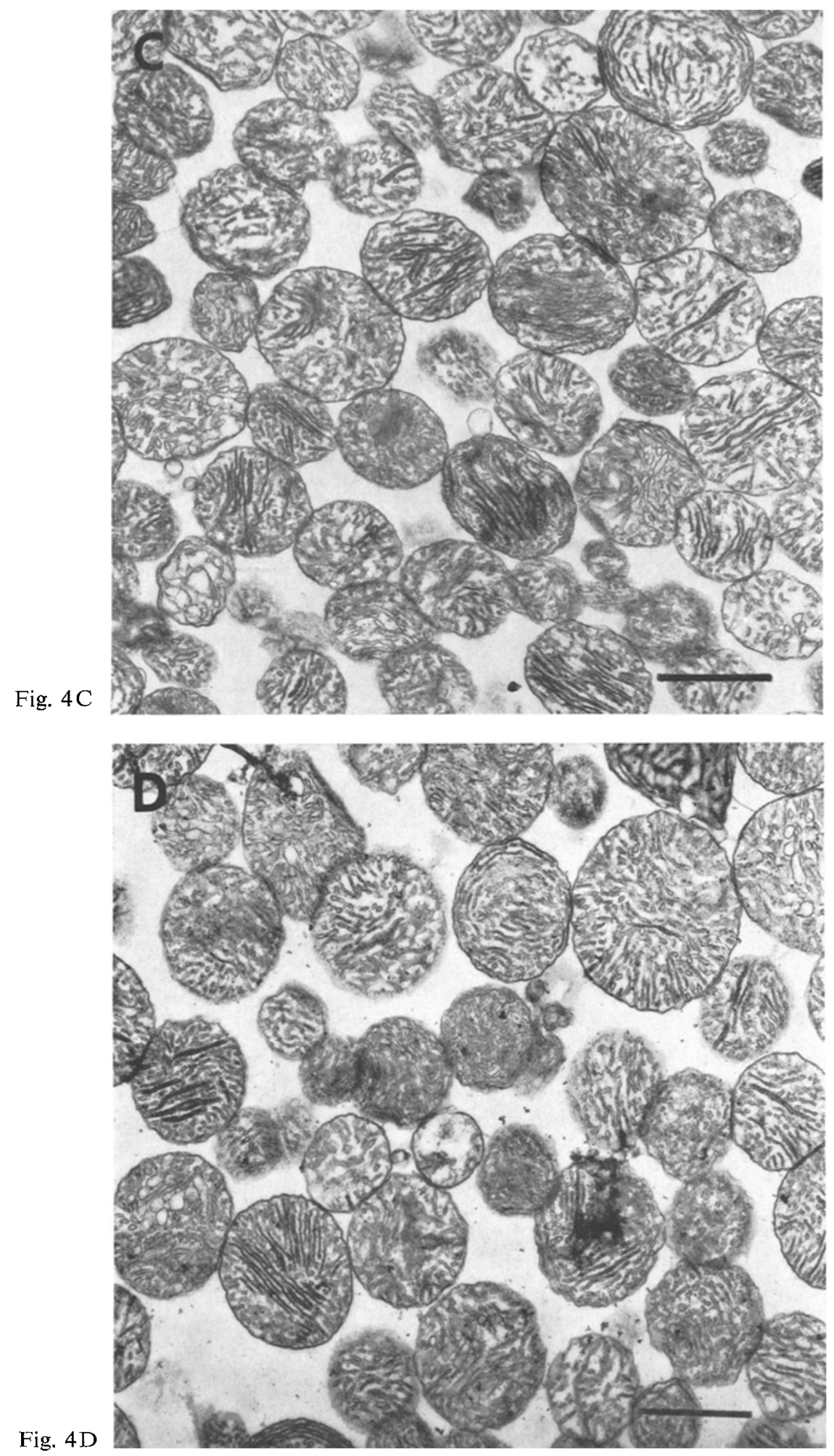

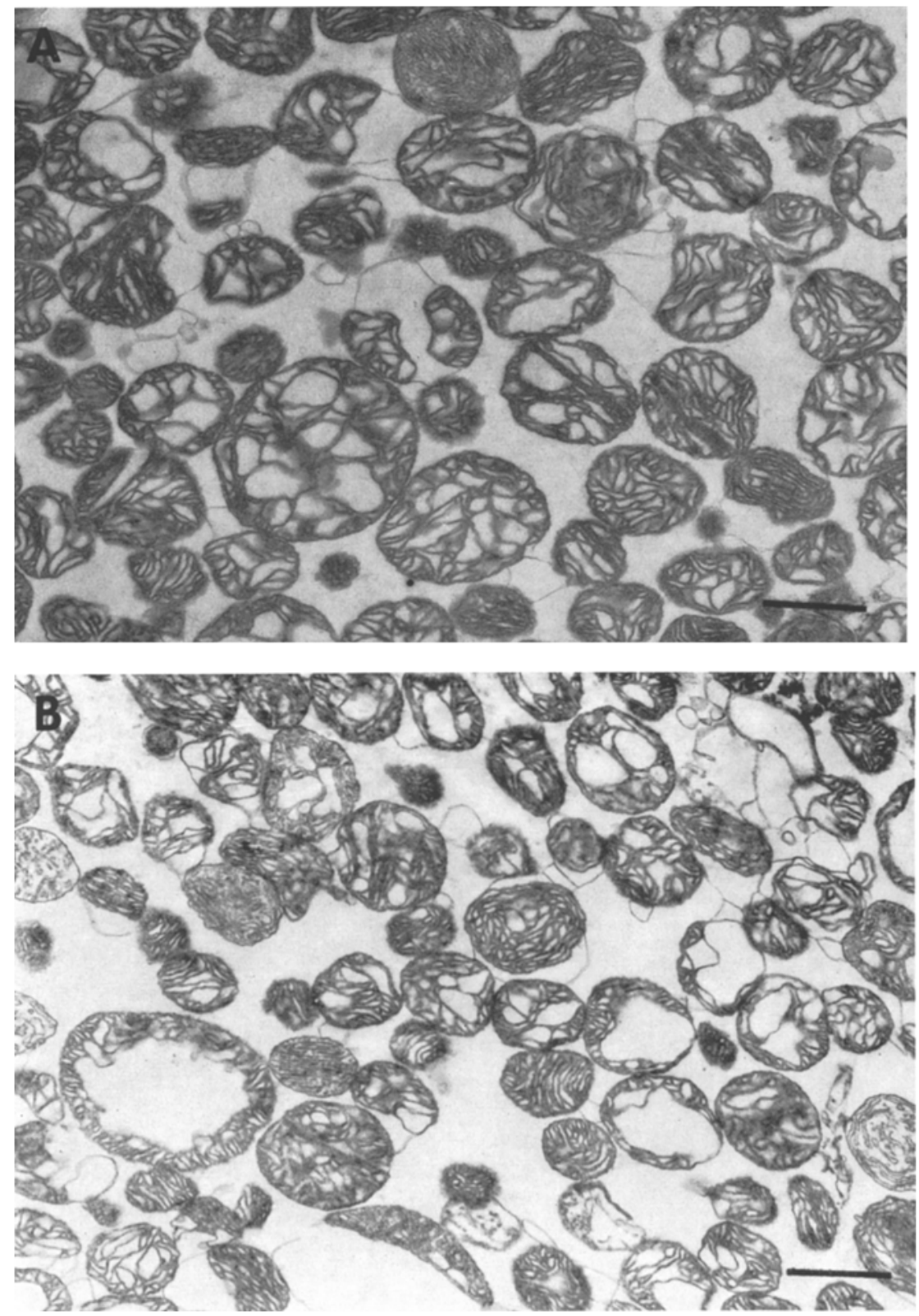

Fig. 5A and B. RMM after 5-min (A) and 20-min (B) incubation in state 2. Note that the mitochondria are in a condensed state in both instances. Scale: $1 \mu . \times 16,000$

At no time were RMM observed in configurations resembling those described by Penniston, Harris, Asai, and Green [13] as "energized twisted" or by Hackenbrock [6] as "maximally condensed". 

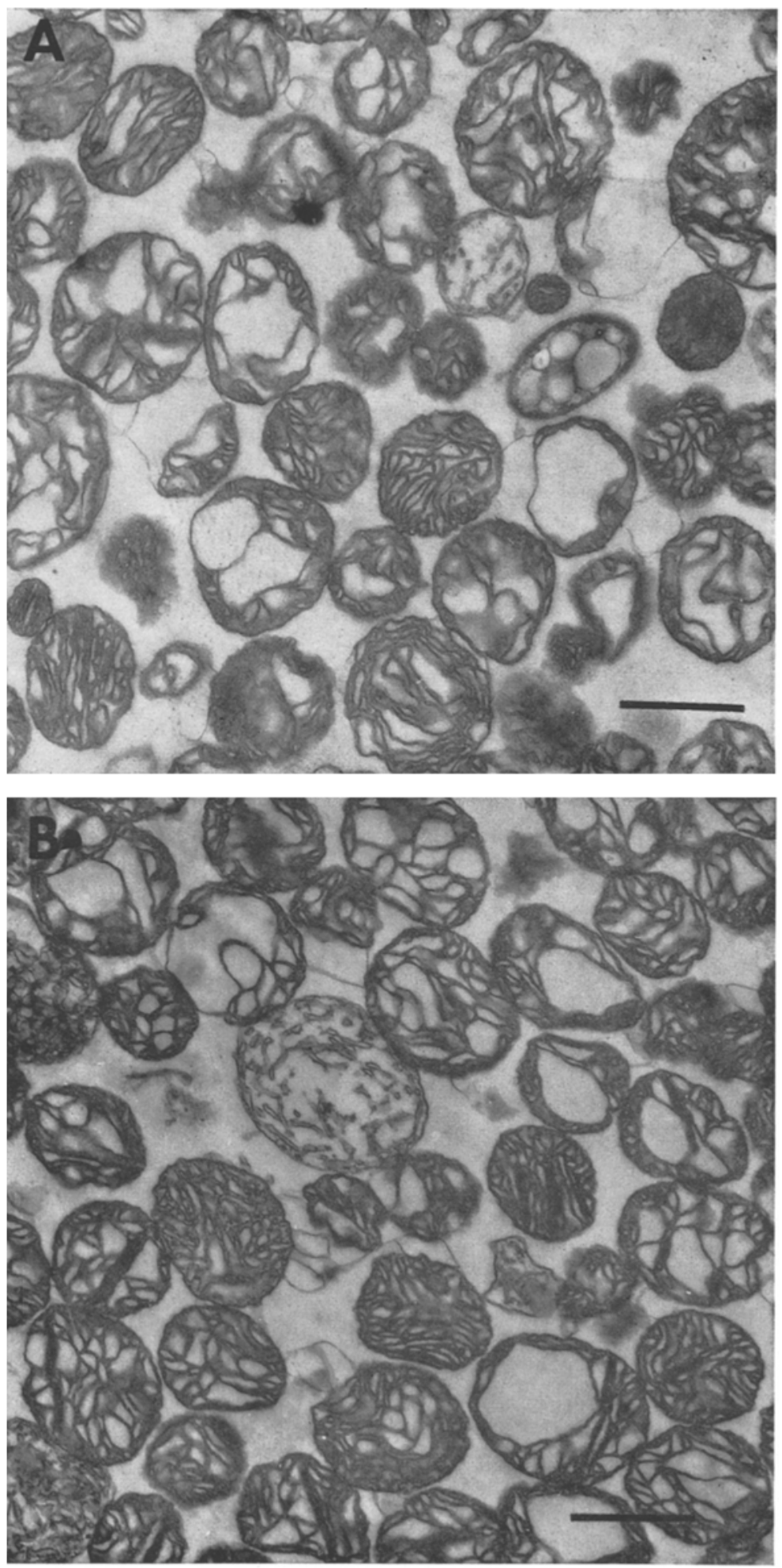

Fig. 6A $-D$. The effect of time, and ADP $(0.42 \mathrm{~mm})$ and DNP $\left(10^{-4} \mathrm{M}\right)$ on RMM after after the addition of ADP (C) or 

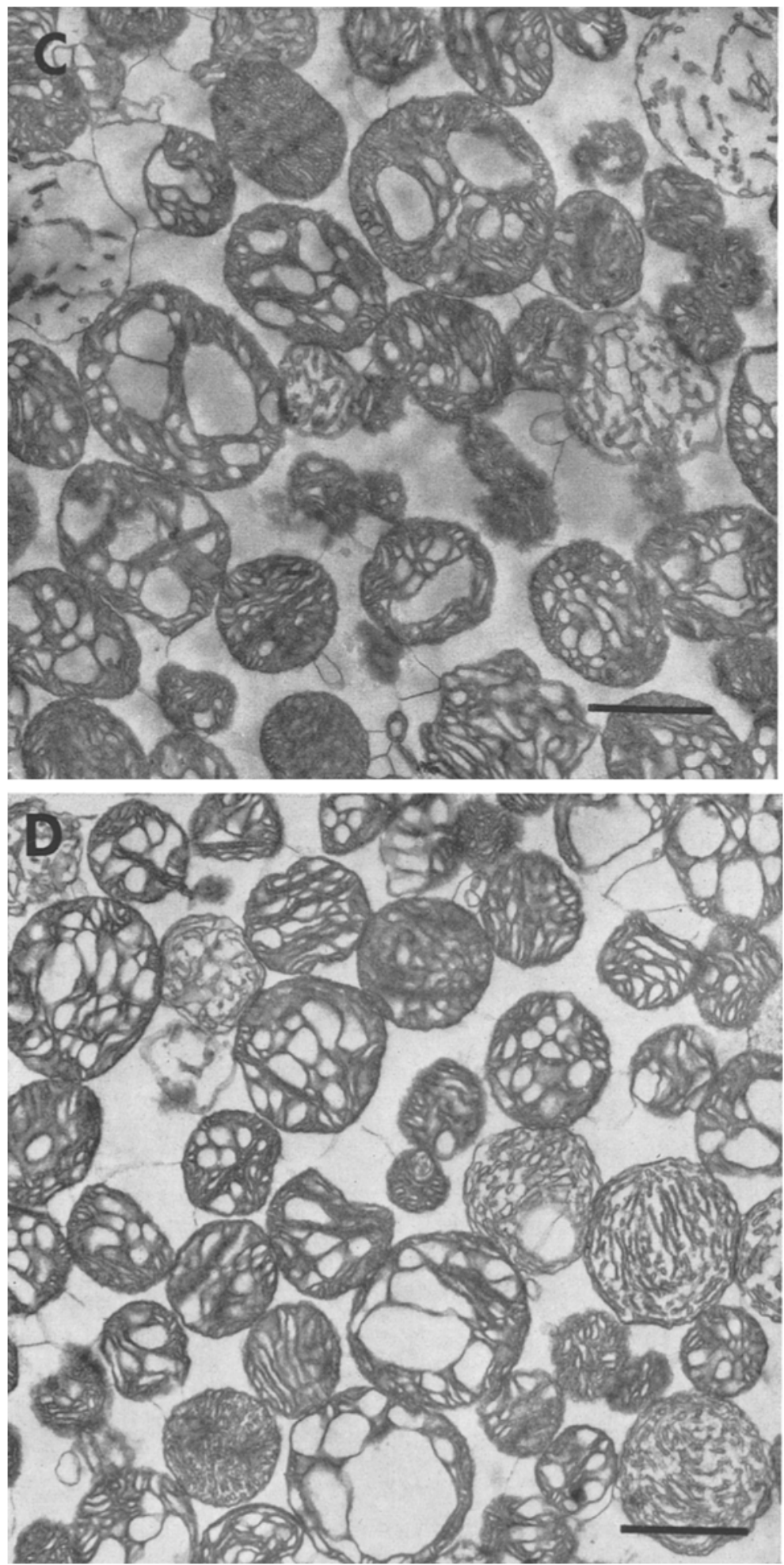

$30 \mathrm{~min}$ in state 4 . RMM were fixed after $5 \mathrm{~min}$ (A) and after $30 \mathrm{~min}$ (B) in state 4 , and DNP (D). Scale: $1 \mu . \times 16,000$ 


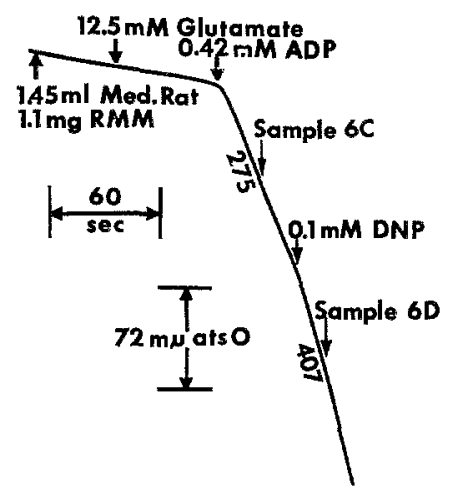

Fig. 6E represents a polarographic tracing of oxygen utilization during sampling of RMM during states 3 and $3 \mathrm{U}$. The electron micrographs resulting from samples obtained in this experiment are shown in $\mathrm{C}$ and $\mathrm{D}$. Conditions of incubation are the same as those described for Fig. 1

\section{Discussion}

At the present time, there exist three major hypotheses which attempt to explain the phenomenon of energy conservation during oxidative phosphorylation catalyzed by the mitochondrial system of enzymes. These are the chemical coupling, chemiosmotic coupling, and conformational coupling models. (For a review of these hypotheses, see [14].) The most recent of these, the conformational coupling model, holds that energy from electrontransfer reactions is initially conserved as potential energy of conformation of components of the inner mitochondrial membrane rather than in a covalent bond as suggested by the chemical coupling model, or as an electrochemical gradient as suggested by the chemiosmotic model. This conformational energy is thought to be conserved ultimately in the terminal phosphate bond of ATP. It is important that the evidence for such a model be tested in as wide a variety of mitochondrial types as possible.

The basis for the conformational coupling hypothesis, i.e., observable ultrastructural changes in the inner mitochondrial membrane, has been under investigation in a number of laboratories. Hackenbrock [6] has demonstrated a correlation between functional and ultrastructural states as determined by electron microscopy and light scattering measurements with rat liver mitochondria. Similar ultrastructural phenomena have been reported by Goyer and Krall [4] in rat kidney mitochondria. Green and his co-workers [5] have also demonstrated ultrastructural-state alterations in association with different functional states in mitochondria isolated from beef, rat, and canary heart tissues. However, Sordahl et al. [15] have recently been unable to demonstrate any generalized relation between 
ultrastructural and biochemical states in heart mitochondria isolated from rats, guinea pigs, rabbits, and beef. The present investigation with rat skeletal muscle mitochondria appears to agree with the conclusions of Sordahl et al. [15].

Hackenbrock [6] reported that rat liver mitochondria undergo a shift from the condensed to the orthodox conformation after short-term (15 min) incubation in state 1 at $30^{\circ} \mathrm{C}$. Furthermore, this orthodox state was not released upon the addition of substrate and ADP. Experimental results of the present investigation have shown a similar shift to the orthodox configuration after 10 -min incubation in state 1 at $25^{\circ} \mathrm{C}$. We were unable to demonstrate any immediate shift from the orthodox configuration upon the addition of substrate, ADP, ATP, or an uncoupler of oxidative phosphorylation. The large population shift from the condensed to the orthodox configuration in liver mitochondria reported by Hackenbrock [6] after 15 min of incubation in state 4 was not observed in this laboratory with skeletal muscle mitochondria even after $30 \mathrm{~min}$ of incubation in state 4 . Furthermore, the addition of ADP or uncoupler to mitochondria after 30-min incubation in state 4 induced no immediately observable shift in mitochondrial ultrastructure even of a qualitative nature (i. $e_{.}$, toward a more- or less-dense matrix). Skeletal muscle mitochondria were capable of undergoing an ultrastructural shift to the orthodox form as was seen in the state 1 incubation.

The results of this investigation would suggest that, in the case of rat skeletal muscle mitochondria, observable ultrastructural alterations are not necessarily concomitant with a readily observable state 4 to state 3 biochemical transition. It may be argued that, if conditions other than those employed in this study were used, perhaps such an ultrastructural shift might be observed. However, one would expect that conditions which would permit a functional transition to be easily demonstrated would also permit ultrastructural alterations to occur, especially if such alterations are basic to energy conservation during oxidative phosphorylation in the mitochondria. We wish to emphasize, however, that the data presented herein do not invalidate the many studies which demonstrate ultrastructural alterations coincident with functional states in mitochondria of various other types of tissues, nor do the studies of Jasper and Bronk [10] or of Harris, Williams, Caldwell, Green, and Valdivia [9] which report such ultrastructural alterations in relatively intact tissues during functional regulation. It is possible that our data represent special conditions of skeletal muscle mitochondria not present in other cell type mitochondria reported to date. 
We wish to acknowledge the skilled technical assistance of Mrs. Mary Lu Hellie. Dr. Louis A. Sordahl kindly provided us with a copy of his manuscript [15] prior to publication.

This work was supported by U. S. Public Health Service grant AM 10056 from the National. Institute of Arthritis and Metabolic Diseases, by a grant from the Office of Research Administration, University of Michigan, and by undergraduate research participation funds from the Department of Zoology.

\section{References}

1. Chance, B., Williams, G. R. 1956. The respiratory chain and oxidative phosphorylation. In: Advances in Enzymology. F. F. Nord, editor., p. 65. Interscience, New York.

2. Estabrook, R. W. 1967. Mitochondrial respiratory control and the polarographic measurement of ADP:O ratios. In: Methods in Enzymology, Vol. 10. R. W. Estabrook and M. E. Pullman, editors, p. 41. Academic Press, New York.

3. Gornall, A. G., Bardawill, C. J., David, M. M. 1949. Determination of serum proteins by means of the biuret reaction. $J$. Biol. Chem. 177:751.

4. Goyer, R. A., Krall, R. 1969. Ultrastructural transformation in mitochondria isolated from kidneys of normal and lead intoxicated rats. J. Cell Biol. 41:393.

5. Green, D. E., Asai, J., Harris, R. A., Penniston, J. T. 1968. Conformational basis of energy transformations in membrane systems. III. Configurational changes in the mitochondrial inner membrane induced by changes in functional states. Arch. Biochem. Biophys. 125:684.

6. Hackenbrock, C. R. 1966. Ultrastructural bases for metabolically linked mechanical activity in mitochondria. I. Reversible ultrastructural changes with change in metabolic steady state in isolated liver mitochondria. J. Cell Biol. 30:269.

7. - 1968. Ultrastructural bases for metabolically linked mechanical activity in mitochondria. II. Electron transport-linked ultrastructural transformations in mitochondria. J. Cell Biol. 37:345.

8. Harris, R. A., Penniston, J. T., Asai, J., Green, D. E. 1968. The conformational basis of energy conservation in membrane systems. II. Correlation between conformational change and functional states. Proc. Nat. Acad. Sci. 59:830.

9. - Williams, C. H., Caldwell, M., Green, D. E., Valdivia, E. 1969. Energized configurations of heart mitochondria in situ. Science 165:700.

10. Jasper, D. K., Bronk, J. R. 1968. Studies on the physiological and structural characteristics of rat intestinal mucosa. J. Cell Biol. 38:277.

11. Makinen, M. W., Lee, C.-P. 1968. Biochemical studies of skeletal muscle mitochondria. I. Microanalysis of cytochrome content, oxidative and phosphorylative activities of mammalian skeletal muscle mitochondria. Arch. Biochem. Biophys. 126:75.

12. Millonig, G. 1961. A modified procedure for lead staining of thin sections. $J$. Biophys. Biochem. Cytol. 11:736.

13. Penniston, J. T., Harris, R. A., Asai, J., Green, D. E. 1968. The conformational basis of energy transformations in membrane systems. I. Conformational changes in mitochondria. Proc. Nat. Acad. Sci. 59:624.

14. Pullman, M. E., Schatz, G. 1967. Mitochondrial oxidations and energy coupling. Ann. Rev. Biochem. 36:539.

15. Sordahl, L. A., Blailock, Z. R., Kraft, G. H., Schwartz, A. 1969. The possible relationship between ultrastructure and biochemical state of heart mitochondria. Arch. Biochem. Biophys. 132:404. 